\title{
ANATOMIA DO FÍGADO E VIAS BILÍFERAS DO MUÇUÃ (Kinosternon scorpioides)
}

\author{
(Anatomy of the liver and bile ducts from the reptile Kinosternon scorpioides)
}

MACHADO JÚNIOR, A.A.N. ${ }^{1}$; SOUSA, A.L. ${ }^{2}$; CARVALHO, M.A.M. ${ }^{1}$; SANTOS, F.C.F. ${ }^{1}$; ALVES, F.R. ${ }^{2}$

1Universidade Federal do Piauí;

2Universidade Estadual do Maranhão.

RESUMO - Foram pesquisados 20 fígados de muçuãs (Kinosternon scorpiodes) adultos obtidos mediante autorização do IBAMA (licença $n \div 006 / 02$ e processo $n=022012001113 / 2002-81$ ). Os animais foram anestesiados com cloridrato de xilazina (1 mg/Kg/lM) e cloridrato de quetamina $(20 \mathrm{mg} / \mathrm{Kg} / \mathrm{IM})$ e após relaxamento muscular, foram sacrificados através da aplicação de tiopental sódico a $2,5 \%$, na dose letal de $60 \mathrm{mg} / \mathrm{Kg} / \mathrm{EV}$. Em seguida prosseguiu-se com a abertura da cavidade pleuroperitoneal e a dissecação do órgão, auxiliada por lupa. Em 100\% dos animais estudados aquele órgão apresentase como um fígado bastante volumoso, de formato retangular, coloração marrom, se estendendo por toda a porção média da cavidade pleuroperitoneal, envolvendo o pâncreas, duodeno e o estômago. Apresenta-se dividido, por fissuras externas, em cinco lobos: lateral e medial direitos, quadrado, lateral esquerdo, e caudato, este com o processo papilar. O fígado encontra-se mantido na posição anatômica pela pressão das vísceras pleuroperitoneais e pelos ligamentos, quais sejam, hepatogástrico (ligandoo ao estômago), hepatoduodenopancreático (ligando-o ao duodeno e pâncreas), hepatopulmonar (ligando-o ao pulmão), hepatopleuroperitoneal (ligando-o à porção ventral da cavidade pleuroperitoneal) e hepatopericárdico (ligando-o ao saco pericárdico). Quanto às vias bilíferas, observa-se que são formadas por ramos oriundos dos lobos hepáticos, que se juntam formando os ductos principais direito e esquerdo, os quais, juntamente com o ducto cístico, oriundo da vesícula biliar, formam o ducto colédoco. De acordo com as observações realizadas sobre a morfologia externa, conclui-se que o fígado do muçuã assemelha-se ao dos principais mamíferos domésticos, no que se refere à forma, número, caracterização e drenagem biliar dos lobos hepáticos, diferindo, entretanto, do das espécies mais próximas, como as salamandras, cobras e outros répteis.

Palavras-chave: Anatomia, fígado, vias bilíferas, Kinosternon scorpioides.

SUMMARY - Twenty livers of adult muçua were analyzed. The samples were obtained through an authorization of IBAMA (license $n^{\circ}=006 / 02$, process $n^{\circ} 0220120011113 / 2002 / 81$ ). The animals were anesthetized with xilazine $(1 \mathrm{mg} / \mathrm{Kg} / \mathrm{lM})$ and ketamine $(20 \mathrm{mg} / \mathrm{Kg} / \mathrm{lM})$. After muscular relaxation, they were sacrificed by means of an injection of sodium thiopental at $2.5 \%$ in the lethal doses of $60 \mathrm{mg} / \mathrm{Kg} / \mathrm{IV}$ ). The coelomatic cavity was opened and the liver dissected with the aid of a magnifying glass. In all the animals the liver was quite voluminous, brown, rectangular in shape, extending through the whole medium portion of the coelomatic cavity, covering the pancreas, duodenum and stomach. External fissures divided the liver in 5 lobes: right lateral and median; square; left lateral and caudate with a papillae process. The liver is maintained in its anatomical position by the pressing of the coelomatic viscera as well as by the following ligaments: hepatogastric (connecting to the stomach); hepatoduodenopancreatic (connecting to the duodenum and pancreas); hepatopulmonar (connecting to the lung); hepatocoelomatic (connecting to the ventral portion of the coelomatic cavity) and hepatopericardic (connecting to the pericardial bursa). In regard to the bile ducts, it is observed that branches originated from the hepatic lobes pull together forming the main right and left ducts, which, together with the cystic duct derived from the gall bladder vesicle, originate the bile duct. According to these observations we concluded that the liver of the muçua is similar to the domestic mammals regarding the shape, number, characterization and bile draining of the hepatic lobe and differs from the ones of the related species such as salamander, snakes and other reptiles.

Key-words: Anatomy, liver, bile duct, Kinosternon scorpioides. 


\section{Introdução}

Diversos trabalhos estão sendo desenvolvidos no campo da ecologia animal, abordando inúmeros aspectos de interesse ecológico, com o intuito de amenizar os problemas em torno da biodiversidade do planeta, evitando assim que determinadas espécies venham se tornar extintas. Assim, aspectos morfológicos, fisiológicos e comportamentais têm assumido papel de elevada importância para a preservação de espécies da nossa fauna, pois tem propiciado ao homem conhecimentos que permitem entender a forma como interagem determinados grupos de animais no ambiente, ou mesmo em regime de semi-confinamento.

Nos últimos anos, no Brasil, tem-se verificado um notável interesse pela criação de animais silvestres, com a organização de criatórios específicos, com potencial para serem explorados na produção de alimentos. Em regiões mais carentes do país, onde é cada vez mais difícil o acesso à proteína animal, temse procurado fontes alternativas de alimentos, através da utilização econômica de animais nativos, dando uma proteína animal de baixo custo e, com a criação em cativeiro, evitar a sua extinção.

O muçuã (Kinosternon scorpioides), pequeno réptil da ordem dos testudines, pertence à família dos kinosternidae e mede de vinte a trinta centímetros de comprimento. É uma tartaruga de água doce, típica das várzeas da região amazônica e chamada no Maranhão de jurará (ENCICLOPÉDIA..., 1999). É uma espécie bastante encontrada que se estende desde o norte dos Estados Unidos até o sul da Argentina e Bolívia (BRAMBLE et al., 1984).

A maioria dos répteis é carnívora e seu sistema digestório não é muito diferente dos anfíbios (VILLEE et al., 1988). Constitui-se de boca, faringe, esôfago, estômago, intestinos delgado e grosso, órgãos anexos, que são as glândulas salivares, pâncreas e fígado, reto e cloaca (BERNARDE, 2003).

Dados relativos às características anatômicas e fisiológicas do aparelho digestório dos répteis, em especial o muçuã, são ainda muito escassos, no entanto algumas informações são fornecidas por BERNARDE (2003), o qual cita que o aparelho digestório tem as funções de receber o alimento digerido, estocá-lo temporariamente, reduzi-lo física e quimicamente, absorver os produtos da digestão, reter temporariamente os restos não digeridos e depois eliminá-los.

A boca, em geral, é grande e uma das características dos répteis é engolir suas presas sem mastigar (FERNANDES, 1981). Os dentes quase sempre estão presentes, faltando, porém, em todos os testudines, onde são substituídos por bainhas córneas que revestem as suas maxilas (KÜKENTAL e MATTHES, 1969).

Em geral, os répteis não aquáticos têm glândulas orais mais desenvolvidas do que os anfíbios. Isto ocorre, devido à necessidade de umidecer o alimento seco para reduzir a fricção durante a deglutição (ORR, 1986).

Depois da curta faringe acha-se o esôfago, um tubo delgado que vai até o estômago. Este compreende uma região esférica ou fúndica e uma porção menor, pilórica, no lado direito, que se continua com o intestino delgado (STORER et al., 2000).

Uma válvula ileocólica separa os intestinos delgado e grosso e um pequeno ceco costuma estar presente no início do intestino grosso, que quase sempre é mais longo que o delgado (VILLEE et al., 1988).

A cloaca é a parte terminal comum para os sistemas digestório, excretor e reprodutor (STORER et al., 2000).

Segundo MACLELLAND (1986); HILDEBRAND (1995); STORER et al. (2000) o fígado da maioria dos vertebrados é formado por dois lobos e situa-se na frente do estômago. Porém, vertebrados como eqüino, cão, suíno e cutias, apresentam o fígado dividido por extensas fissuras, delimitando os lobos hepáticos em números que variam de cinco a seis, dependendo da espécie animal (GETTY, 1986; MENEZES et al., 2001).

O fígado, a maior glândula do organismo, possui funções múltiplas e complexas. Representa o local de numerosos processos metabólicos, pois recebe todo material absorvido ao nível dos intestinos, exceção feita a certa quantidade de lipídios transportados por via linfática. Devido a essas importantes mediações o órgão apresenta-se numa 
situação de grande destaque no organismo, já que metaboliza, armazena, sintetiza e elimina substâncias absorvidas. Tal fato se processa pela bile, secreção exócrina da célula hepática, de enorme importância na digestão e absorção de gorduras (POUGH et al., 1999). Devido a essas características, numerosos são os pesquisadores preocupados em estudar os múltiplos aspectos macroscópicos desta complexa glândula, tanto no homem quanto nos animais.

No fígado dos vertebrados existe uma rede de finos túbulos biliares, que reúnem as secreções celulares, a bile, em um ou mais ductos hepáticos que drenam o fígado. Mais próximo ao intestino estes ductos juntam-se ao ducto cístico, ligando-o à vesícula biliar na qual a bile pode ser armazenada. Após esta junção, tem-se um ducto biliar comum (ducto colédoco) que se abre no intestino delgado (MESSER, 1938; HICKMAN, 1967; BAROUDI, 1970; ROMER e PARSONS, 1985; DYCE et al., 1997; FOWLER e MILLER, 1999), com exceção das lampreias, peixes teleósteos e alguns herbívoros, como o cavalo, os quais não apresentam vesícula biliar, sendo o seu produto (bile) lançado diretamente no duodeno (HILDEBRAND, 1995).

Sobre a distribuição dos ductos biliares no parênquima hepático do muçuã, nenhum dado foi obtido na literatura consultada, porém, considerando os vertebrados de um modo geral, a literatura específica sobre as vias bilíferas é vasta. Dentre os autores que se preocuparam com este aspecto, pode-se destacar, nos bovinos (BASTOS NETO e PRADA, 1983; SOUZA, 1984); na anta (Tapirus americanus), MIGLINO et al. (1982); no quati (Nasua narica), SOUZA et al. (1982); no equino (MARÇAL et al. 1993); nos muares (MAXIMIANO NETO et al. 2000); no tamanduá bandeira (Myrmecophaga tridactila), SOUZA et al. (1986) e no cateto (Tayassu tajacu), MIGLINO et al. (1986).

OLIVEIRA et al. (1977), estudando a sistematização das vias bilíferas na cobaia (Cavia porcellus), verificaram que o ducto colédoco destes animais é formado por junção dos ramos: papilar, principal esquerdo e principal direito; papilar e união do principal direito e esquerdo; principal esquerdo e principal direito; papilar, principal esquerdo, principal direito e lateral direito; papilar, lateral esquerdo, principal esquerdo e principal direito; papilar, principal esquerdo e dos ramos que trazem a bile do lado direito do fígado, sendo um constituído pela junção do ramo lateral direito e do medial direito e outro formado por vários ramos laterais direito e pelo caudato; pelos ramos papilar, principal esquerdo, medial direito, e principal direito; papilar, principal esquerdo, principal direito e caudato; papilar, principal esquerdo, principal direito e dos laterais esquerdos; de um único ducto formado pela junção dos ramos principal direito e principal esquerdo; de um ducto formado pelos ramos principal direito e principal esquerdo, unido ao papilar; dos ramos principal direito, principal esquerdo e medial direito; de um ramo formado pelo principal direito e principal esquerdo e de outro formado pelos ramos lateral direito e papilar; dos ramos principal direito, principal esquerdo e união do papilar e lateral direito; ramos principal esquerdo, intermédio, principal direito e união do papilar e caudato; ramos principal esquerdo, lateral esquerdo, papilar, medial esquerdo, intermédio, principal direito e caudato.

De acordo com RAYS et al. (1980), o rumus principalis dexter do fígado de cobaias (Cavia porcellus) mostrou-se livre de tributários em 7 preparações (18\%). Em $82 \%$ das preparações os afluentes vinham sempre dos lobus dexter lateralis, lobus dexter medialis e lobus caudatus (processus caudatus), sendo exclusivamente formado por eles em cerca de $41 \%$ delas. Juntamente com os ramos desses 3 lobos aparecem, em $29 \%$ das preparações, o ramus processi papillaris. Em dois casos (4\%), o ramus principalis dexter chega ao ramus principalis sinister. Formam-se ainda coletores biliares do fígado que se mostram independentes do ramus principalis dexter: o ramus processi papillaris $(11 \%)$, o ramus processi caudati $(7 \%)$, o ramus lobi dextri medialis (4\%), um tronco formado pelos ramus lobi dextri medialis e ramus dorsalis lobi dextri lateralis. O ramus dorsalis lobi dexter lateralis, o ramus dorsomedialis lobi dextri lateralis, o ramus ventromedialis lobi dextri lateralis, o ramus ventralis lobi dextri lateralis, o ramus lobi dextri medialis, o ramus processis papillares e o ramus 
processis papillaris e o ramus processi caudati drenam como coletores principais, as regiões dorsal, dorsomedial, ventromedial e ventral do lobus dexter lateralis, o lobus dexter medialis, - lobus caudatus (processus papillaris e processus caudatus) respectivamente.

PRADA et al. (1981), pesquisando o sistema excretor do fígado do ratão do banhado (Miocastor coipus), concluiram que: o ductus choledocus, livre de tributários em todas as peças, mostra-se constituído pela união do ramus principalis dexter e ramus principalis sinister $(60,0 \%)$, pela triconvergência desses dois ramos e ductus cysticus $(20,0 \%)$ e pela confluência do ductus hepaticus mais ductus cysticus $(20,0 \%)$; o ductus hepaticus é identificado em $20,0 \%$, exibindo-se livre de afluentes, resultando sempre da associação do ramus principalis dexter e ramus principalis sinister; o ductus cysticus integra o sistema do ramus principalis sinister (33,3\%) e o sistema do ramus principalis dexter (26,7\%) e associa-se aos dois citados ramos, por triconvergência $(20,0 \%)$ e ao ductus hepaticus (20,0\%); integra o sistema do ramus principalis dexter, o ramus processi caudati, o ramus lobi dextri lateralis, o ramus lobi dextri medialis e, por vezes, o ductus cysticus, além de numerosas contribuições procedentes do lobus dexter lateralis, lobus dexter medialis e lobus caudatus (pars supraportalis, processus papillaris e processus caudatus); participam do sistema do ramus principalis sinister, o ramus lobi sinistri lateralis, o ramus lobi sinistri medialis, o ramis lobi quadratie, por vezes, o ductus cysticus, afora numerosas contribuições vindas do lobus sinister lateralis, lobus sinister medialis, lobus quadratus, lobus caudatus (pars supraportalis e processus papillaris) e lobus dexter medialis.

Desta forma, tornam-se relevantes estudos sobre a anatomia dos órgãos digestivos, para embasar o manejo nutricional desses animais. A presente pesquisa objetivou estudar o fígado e vias bilíferas do muçuã (kinosternon scorpiodes).

\section{Material e Métodos}

Foram estudados 10 exemplares adultos de $K$. scorpioides para relato da anatomia do fígado e das vias bilíferas. Os animais foram adquiridos mediante autorização do Instituto
Brasileiro do Meio Ambiente e dos Recursos Renováveis (IBAMA), de acordo com a licença no006/02 e processo oㅜ 022012001113/200281 , devendo-se ressaltar que estes animais geralmente apresentavam-se mutilados, o que inviabilizava a sua reintrodução na natureza.

Os animais foram anestesiados com cloridrato de xilazina $(1 \mathrm{mg} / \mathrm{Kg})$ e cloridrato de quetamina $(20 \mathrm{mg} / \mathrm{Kg})$ por via intramuscular, segundo protocolo de THURMON et al. (1996). Após relaxamento, os animais foram sacrificados com a aplicação de dose letal $(60$ $\mathrm{mg} / \mathrm{Kg}$ ) de tiopental sódico a 2,5\%, por via endovenosa, para remoção do plastrão.

Com o uso de uma serra de aço se procedia a desarticulação da ponte óssea que une o plastrão à carapaça e, com auxílio de bisturi, removiam-se os tecidos e musculatura para o acesso à cavidade pleuroperitoneal, observando então a topografia dos órgãos a fresco. Nessa oportunidade, no fígado foi injetado látex, através da colédoco, para facilitar a dissecação das vias bilíferas.

As peças foram fixadas em solução aquosa de formaldeído a $10 \%$ por um período mínimo de 24 horas, para posterior dissecção macroscópica do fígado e vias bilíferas, com o auxílio de lupa circular de luz fria.

\section{Resultados e Discussão}

Em $100 \%$ dos animais estudados o fígado apresenta-se como um órgão bastante volumoso, com formato retangular e de coloração marrom, variando entre os tons claro e escuro, preenchendo toda a porção mediana da cavidade pleuroperitoneal, envolvendo o pâncreas, duodeno e o estômago, à semelhante do que foi descrito por KÜKENTAL e MATTHES (1969) para a salamandra Lacerta agilis, por ROMER e PARSONS (1985), de forma genérica, aos vertebrados, e por POUGH et al. (1999) para as tartarugas. Os dados do K. scorpioides somente diferiram do relatado por FOWLER e MILLER (1999) para as cobras cujo fígado possui um formato alongado e elíptico.

O fígado do muçuã ( $K$. scorpioides), na totalidade dos animais estudados, apresentase constituído de cinco lobos sendo dois à esquerda e três à direita do plano mediano. Tal 
achado difere do descrito por KÜKENTAL e MATTHES (1969), para Lacerta agilis, por HILDEBRAND (1995), para a maioria dos vertebrados, por MACLELLAND et al. (1986), para as aves, e STORER et al. (2000) para os répteis, quando citam o fígado destes animais formado apenas por dois lobos, um direito e um esquerdo, assim como MENEZES et al. (2001) para as cutias, as quais apresentam o fígado dividido em seis lobos. Assemelha-se, entretanto, ao descrito por GETTY (1986) para o fígado do eqüino, que também se encontra dividido em cinco lobos.

O lado esquerdo encontra-se formado pelos lobos lateral esquerdo e caudato, com seu processo papilar. É neste lado que encontramos ainda, inserido entre esses lobos, o estômago, fazendo íntimo contato com o fígado (FIGURA 2). Isto diferindo do descrito por STORER et al. (2000), para a maioria dos répteis, onde o fígado encontra-se localizado cranial ao estômago. O lado direito, por sua vez, encontrase dividido em três lobos: o lateral, o e medial direito e o quadrado (FIGURA 1).

FIGURA 1 - VISTA DA PARIETAL DO FÍGADO DO $K$. scorpioides FORA DA CAVIDADE, EM PEÇA FIXADA, MOSTRANDO SUAS DIVISÕES EM LOBOS LATERAL ESQUERDO (1), PROCESSO PAPILAR DO LOBO CAUDATO (2), LATERAL (3) E MEDIAL DIREITO (4) E QUADRADO (5). É POSSÍVEL OBSERVAR AINDA PARTE DA VESÍCULA BILIAR (SETA BRANCA) E ESTÔMAGO (SETAAZUL).

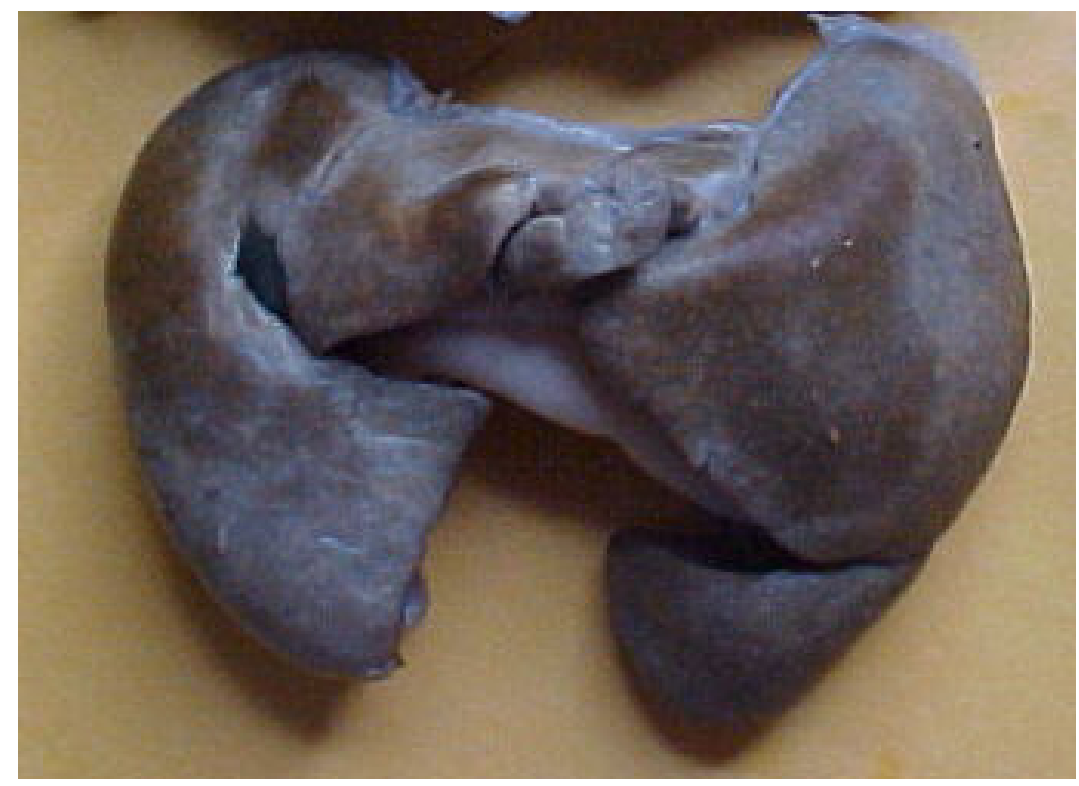

Pela face visceral, entre os lobos lateral e medial direito, encontramos a vesicular biliar, fortemente fixada a estes lobos, ultrapassando, no entanto, a margem caudal do lobo direito. Este órgão armazena a bile, secreção hepática de coloração verde escuro, que é lançada diretamente no duodeno (FIGURA 2). Tal achado difere do relatado por FOWLER e MILLER (1999) para as cobras, cuja vesícula biliar apresenta-se separada do fígado, e do descrito por GETTY (1986), para o cavalo, o qual não apresenta vesícula biliar, sendo a bile lançada diretamente no duodeno.

O fígado está revestido por uma cápsula de tecido conjuntivo e apresenta duas superfícies: uma parietal, voltada para o plastrão, e outra visceral ligada à carapaça. A maior parte da superfície parietal, convexa e lisa, está próxima da parede ventrolateral da cavidade pleuroperitoneal. A parte cranial dos lobos medial direito, lateral esquerdo e quadrado é côncava e relaciona-se com o ápice do coração. A superfície visceral é irregular, côncava e possui a impressão das vísceras, sendo elas, estômago, intestinos e, ainda, ovidutos e ovários nas fêmeas. Tal achado é semelhante ao relatado por MACLELLAND (1986) para as aves. 
FIGURA 2 - VISTA VENTRAL DA CAVIDADE PLEUROPERITONEAL DO K. scorpioides, MOSTRANDO O LOBO DIREITO SUBDIVIDIDO EM LATERAL DIREITO (1), MEDIAL DIREITO (2) E QUADRADO (3); EVIDENCIANDO AINDAA VESÍCULA BILIAR (SETA) ENVOLVIDA PELOS LOBOS LATERAL E MEDIAL DIREITOS.

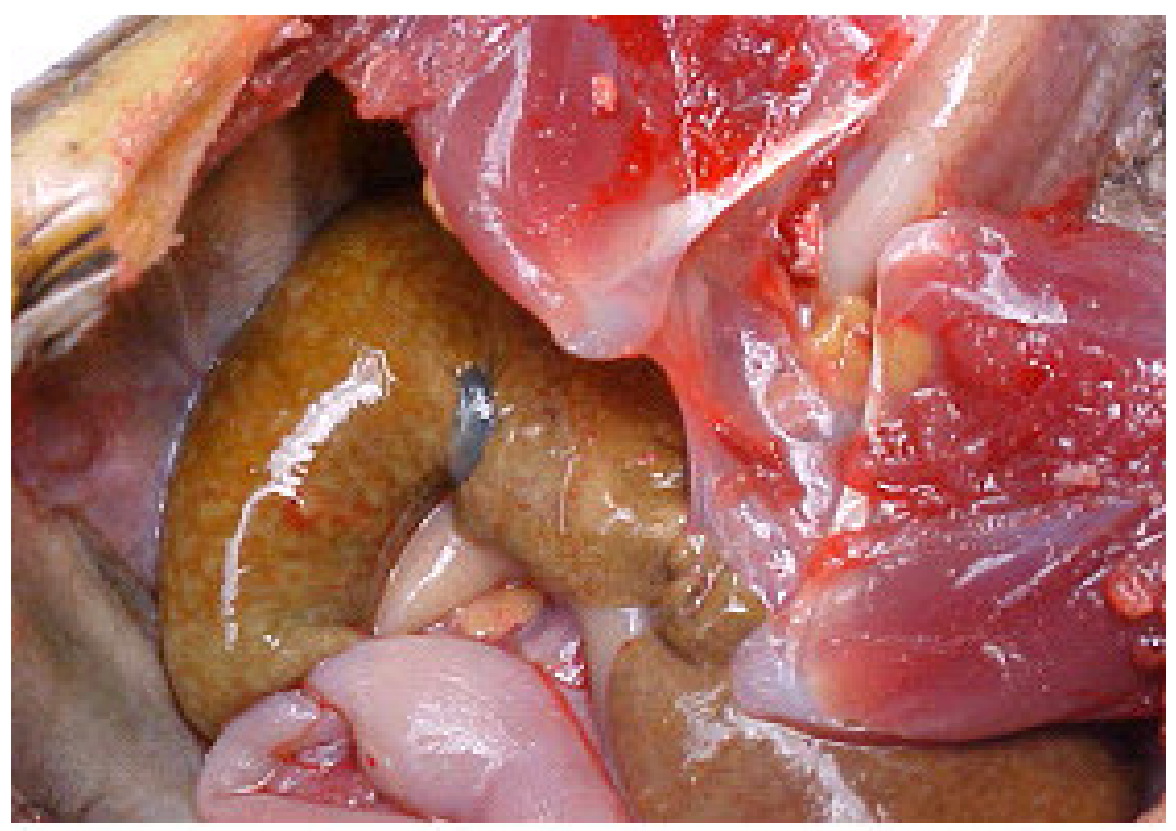

O fígado encontra-se mantido na sua posição anatômica devido à pressão das vísceras pleuroperitoneais (FIGURA 3) e pela presença de ligamentos, auxiliando também nesta função. Entre esses ligamentos temos, o hepatogástrico, ligando o fígado ao estômago, o hepatoduodenopancreático, ligando o fígado ao duodeno e pâncreas, o hepatopulmonar, ligando o fígado ao pulmão, o hepatopleuroperitoneal, ligando o fígado à porção ventral da cavidade peluroperitoneal e o hepatopericárdico, ligando o fígado ao saco pericárdico.

Quanto à topografia, temos o fígado relacionando-se com os pulmões, cranialmente, como o coração craniomedialmente, estômago, ovidutos e ovários caudalmente, intestino delgado caudomedialmente, teto da cavidade pleuroperitoneal dorsolateralmente e assoalho da cavidade ventralmente (FIGURA 3).

A vesícula biliar do $K$. scorpioides é um órgão arredondado, cuja função é armazenar a bile drenada dos lobos hepáticos. Observa-se que este tipo de drenagem e sua disposição anatômica é semelhante aos mamíferos domésticos, entretanto difere em alguns aspectos do que foi descrito por MACLELLAND (1986) e DYCE et al. (1997) para as aves, as quais apresentam apenas o lado direito do fígado drenando sua bile para a vesícula biliar, sendo que o lado esquerdo lança seu produto diretamente no duodeno, e do descrito por ROMER e PARSONS (1985); GETTY (1986); HILDEBRAND (1995), para as lampreias, peixes teleósteos e alguns herbívoros, como o cavalo, os quais não apresentam vesícula biliar, sendo o seu produto (bile) lançado diretamente no duodeno. 
FIGURA 3 - VISTA VENTRAL DA CAVIDADE PLEUROPERITONEAL DO K. scorpioides, MOSTRANDO O FÍGADO (1) MANTIDO NA SUA POSIÇÃO PELA PRESSÃO DAS VÍSCERAS, ENTRE ELAS O ESTÔMAGO, CAUDALMENTE E À ESQUERDA (2), INTESTINO DELGADO CAUDOMEDIALMENTE (3), PULMÃO CRANIALMENTE (4) E OVÁRIOS (5) E OVIDUTOS CAUDALMENTE (6).

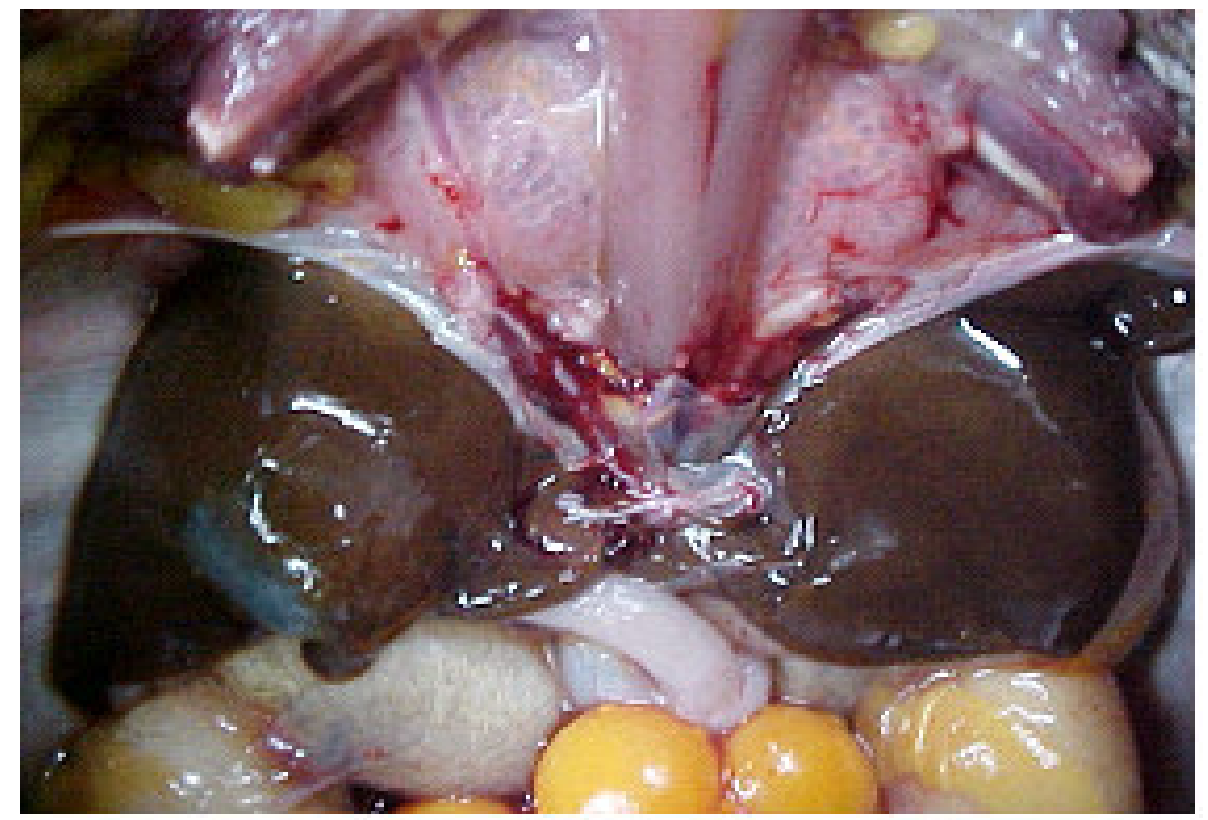

Estes ductos biliares, que drenam a bile dos lobos hepáticos, se reúnem formando os ductos principais direito e esquerdo, que juntamente com o ducto cístico, formam o ducto colédoco, que se abre na porção inicial do duodeno (FIGURA 4). Estes achados assemelham-se aos descritos por MESSER (1938), HICKMAN (1967) e BAROUDI (1970) para os répteis, e por ROMER e PARSONS (1985) e HILDEBRAND (1995) para a maioria dos vertebrados, DYCE et al. (1997) para alguns mamíferos e PRADA et al. (1981) para o ratão do banhado. Diferindo, porém, dos relatos de OLIVEIRA et al (1977) e RAYS et al. (1980), para a cobaia, que apresenta o colédoco formado pela junção dos ramos: papilar, principal esquerdo e principal direito; papilar e união do principal direito e esquerdo; principal esquerdo e principal direito; papilar, principal esquerdo, principal direito e lateral direito; papilar, lateral esquerdo, principal esquerdo e principal direito; papilar, principal esquerdo e dos ramos que trazem a bile do lado direito do fígado, sendo um constituído pela junção do ramo lateral direito e do medial direito e outro formado por vários ramos laterais direito e pelo caudato; pelos ramos papilar, principal esquerdo, medial direito e principal direito; papilar, principal esquerdo, principal direito e caudato; papilar, principal esquerdo, principal direito e dos laterais esquerdos; de um único ducto formado pela junção dos ramos principal direito e principal esquerdo; de um ducto formado pelos ramos principal direito e principal esquerdo unido ao papilar; dos ramos principal direito, principal esquerdo e medial direito; de um ramo formado pelo principal direito e principal esquerdo e de outro formado pelos ramos lateral direito e papilar; dos ramos principal direito, principal esquerdo e união do papilar e lateral direito; ramos principal esquerdo, intermédio, principal direito e união do papilar e caudato; ramos principal esquerdo, lateral esquerdo, papilar, medial esquerdo, intermédio, principal direito e caudato.

Quanto ao arranjo dos ductos bilíferos no parênquima do órgão observa-se que: o ramo principal direito é constituído por afluentes vindos dos lobos medial direito, lateral direito e quadrado, enquanto que o ramo principal esquerdo é formado pelos ductos oriundos dos lobos lateral esquerdo e caudato. 
FIGURA 4 - FÍGADO DO $K$. scorpioides, EM PEÇA FIXADA, MOSTRANDO A VESÍCULA BILIAR (1) PREENCHIDA DE CONTRASTE, MOSTRANDO O TRAJETO DO DUCTO BILIAR PRINCIPAL ESQUERDO (SETA), COLÉDOCO (2) E DUODENO (3).

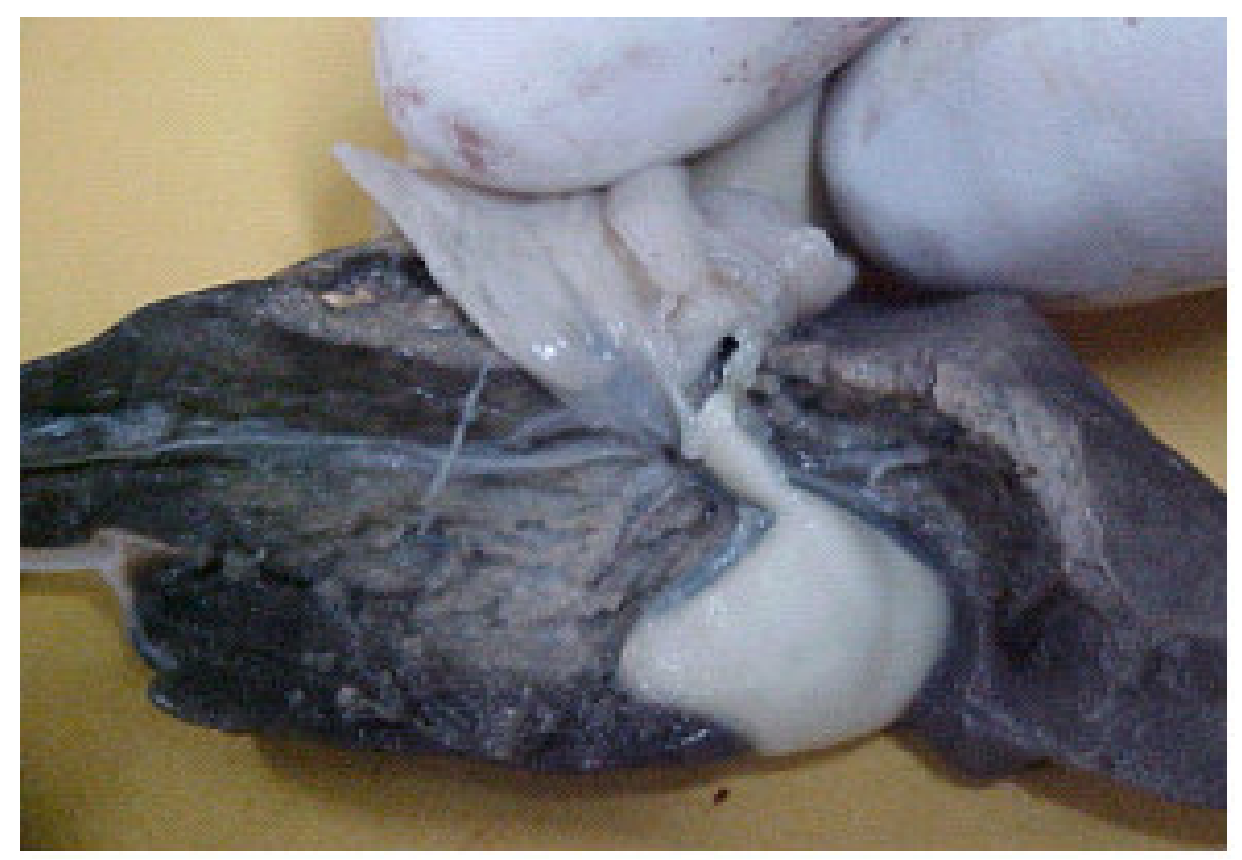

\section{Conclusão}

Diante do exposto, e sob as condições de realização desta pesquisa, pode-se inferir que o fígado do muçuã (Kinosternon scorpiodes) assemelha-se, anatomicamente, ao dos principais mamíferos domésticos, no que se refere à forma, número, caracterização e drenagem biliar dos lobos hepáticos, diferindo, entretanto, do observado em espécies mais próximas, como as salamandras, cobras e outros répteis.

\section{Agradecimentos}

Ao Conselho Nacional de Desenvolvimento Científico e Tecnológico - CNPq pelo apoio financeiro concedido.

\section{REFERÊNCIAS}

BAROUDI, R. Elementos de Zoologia. 6. ed. São Paulo: Nobel, 1970. p. 125-152.

BASTOS NETO, I.P; PRADA, I.L.S. Contribuição das vias bilíferas em bovinos azebuados. Sistematização do ramus principalis sinister. Revista da Faculdade de Medicina Veterinária e Zootecnia da Universidade de São Paulo, São Paulo, v. 20, n. 1, p. 23-27, 1983.
BERNARDE, P.S. Sistema digestório dos répteis. Disponível em: <httpllwww. herpetofauna. hpg.ig. com.br/Pages/SistemaDigestório>. Acesso em: 07 ago. 2003.

BRAMBLE, D.M.; HUTCHISON, J.H.; LEGLER, J.M. Kinosternid shell kinesis: structure, funtion and evolution. Copeia, Lawrence, n. 2, p. 456-475, 1984.

DYCE, K.M.; SACK, W.O.; WENSING, C.I.G. Tratado de anatomia veterinária. Rio de Janeiro: Guanabara Koogan, 1997. p. 645-647.

ENCICLOPÉDIA Britânica do Brasil. Nova Enciclopédia Barsa. São Paulo: Melhoramentos, 1999. v.10, p. 193 , v.12, p. $150,305-307$.

FERNANDES, V. Zoologia. 7. ed. São Paulo: Editora Pedagógica e Universitária, 1981.

FOWLER, M.E.; MILLER, R.E. Zoo \& wild animal medicine: current therapy. 4th ed. Philadelfia: W. B. Saunders Company, 1999. p. 243-248.

GETTY, R. Anatomia dos animais domésticos. 5.ed. Rio de Janeiro: Guanabara, 1986. 1134 p. v.1. HICKMAN, C.P. Princípios de zoologia. Barcelonaa: Ariel, 1967. $505 \mathrm{p}$.

HILDEBRAND, M. Análise da estrutura dos vertebrados. São Paulo: Atheneu, 1995. p. 323-328. 
KÜKENTAL, W.; MATTHES, E. Guia de trabalhos práticos de zoologia. 15. ed. Rio de Janeiro: Atlântida Editora, 1969. p. 391-409.

MACLELLAND, J. Aparelho digestório das aves. In: GETTY, R. Anatomia dos animais domésticos. 5. ed. Rio de Janeiro: Guanabara Koogan, 1986. p. 1813-1828.

MARÇAL, A.V.; FERREIRA, N.; FERNANDES FILHO, A.; STOPIGLIA, A.J. Contribuição ao estudo das vias bilíferas em equinos da raça puro sangue inglês. Sistematização dos ductus principalis sinister. Revista Brasileira de Ciências Morfológicas, São Paulo, v. 10, n. 1, p. 22-27, 1993.

MAXIMIANO NETO, A.; SANTOS, A.L.Q.; MOURA, C.R.; TUNALA, V. Sistematização do ductus principalis sinister em fígados de muares (Equus asinos $x$ Equus caballus). Brazilian Journal of Morphological Sciences, Goiânia, v. 17, p. 235, 2000.

MENEZES, D.J.A.; CARVALHO, M.A.M.; CAVALCANTE FILHO, M.F.; SOUZA, W.M. Configuração do Sistema Venoso Portal na Cutia (Dasyprocta aguti; RODENTIA). Brazilian Journal of Veterinary Research Animal Science, São Paulo, v. 38, n. 6, p. 8, 2001.

MESSER, H.M. An introdution to vertebrate anatomy. New York: The Macmillan Company, 1938. p. 206-208.

MIGLINO, M.A.; PRADA, I.L.S.; D'ERRICO, A.A.; SOUZA, W.M. Contribuição ao estudo do Sistema excretor do fígado em animais silvestres. Vias bilíferas da Anta (Tapirus americanus). In: SEMANA DE VETERINÁRIA, 1., 1982, São Paulo. Anais... São Paulo, 1982. p. 15.

MIGLINO, M.A.; SOUSA, W.M.; NASCIMENTO, A.A.; ARANTES, I.G.; CARVALHAL, R. Contribuição do sistema excretor do fígado em animais silvestres. Vias bilíferas do cateto (Tayassu tayassu). In: CONGRESSO BRASILEIRO DE MEDICINA VETERINÁRIA, 20., 1986, Cuiabá. Anais... Cuiabá, 1986. p. 342.

OLIVEIRA, M.C.; ABREU, M.A.F.; SILVA, P.P.; DEFINE, R.M. Contribuição ao estudo das vias bilíferas na cobaia (Cavia porcellus). In: CONFERÊNCIA ANUAL. SOCIEDADE PAULISTA DE MEDICINA VETERINÁRIA, 32., 1977, Pirassununga. Comunicações Científicas. Pirassununga, 1977. p. 17.

Recebido para publicação: 04/04/2005 Aprovado:
ORR, R.T. Biologia dos vertebrados. 5. ed. São Paulo: Livraria Roca, 1986. p. 95-128.

POUGH, F.H.; HEISER, J.B.; McFARLAND, W.N. A vida dos vertebrados. 2. ed. São Paulo: Atheneu Editora, 1999. p. 86-88.

PRADA, I.L.S.; D'ERRICO, A.A.; BORELLI, V.; SOGORB, S.F. Contribuição ao estudo do sistema excretor do fígado em animais silvestres. Vias bilíferas do Ratão do banhado (Miocastor coypus). In: CONGRESSO BRASILEIRO DE ANATOMIA,13., CONGRESSO LUSO-BRASILEIRO DE ANATOMIA, 4., 1981, Belo Horizonte. Resumos... Belo Horizonte: Sociedade Brasileira de Anatomia, 1981. p. 185-86.

RAYS, M.A.A.; DAYOUB, M.C.O.; SILVA, P.P. Contribuição ao estudo das vias biliares na cobaia (Cavia porcellus). Sistematização do ramus principalis dexter. In: ENCONTRO DE PESQUISAS VETERINÁRIAS, 5., 1980, Ribeirão Preto. Resumos. Ribeirão Preto,1980. p. 33-4.

ROMER, A.S., PARSONS, T.S. Anatomia comparada dos vertebrados. São Paulo: Atheneu, 1985. p. 338-358.

SOUZA, W.M.; PRADA, I.L.S.; D'ERRICO, A.A.; MIGLINO, M.A. Contribuição ao sistema excretor do fígado em animais silvestres. Vias bilíferas do Coati (Nasua narica). In: SEMANA DE VETERINÁRIA, 1., 1982, São Paulo. Anais... São Paulo, 1982. p. 18.

SOUZA, W.M. Contribuição ao estudo das vias bilíferas intra e extra-hepática em bovinos da raça nelore. In: CONGRESSO DE LA ASOCIACION PANAMERICANA DE ANATOMIA, 7., ASOCIACION RIOPLATENSE DE ANATOMIA, 21., 1984, Punta del Este. Anais... Punta del Este: Sociedade brasileira de Anatomia, 1984. p. 159.

SOUZA, W.M.; MIGLINO, M.A.; PRADA, I.L.S.; BORELLI, V. Contribuição ao estudo do sistema excretor do fígado em animais silvestres. Vias bilíferas do Tamanduá bandeira (Myrmecophaga tridactula). In: CONGRESSO BRASILEIRO DE ANATOMIA, 14., 1985, Vitória. Anais... Vitória, Sociedade Brasileira de Anatomia, 1986. p. 80.

STORER, T.I., USINGER, R.L., STEBBINS, R.C., NYBAKKEN, J.W. Zoologia geral. 6. ed. São Paulo: Companhia Editora Nacional, 2000. v. 8, p. 642-654.

THURMON, J.C.; TRANQUILLI, W.J.; BENSON, G.J. Lumb \& jones' veterinary anesthesia. 3rd ed. Baltimore: Lippincott Williams \& Wilkins, 1996. p. 670-685.

VILLEE, C.A.; WALKER Jr., W.F.; BARNES, R.D. Zoologia geral. 6. ed. Rio de Janeiro: Editora Guanabara. 1988. p. 547-557. 